\title{
Perturbações do Comportamento Alimentar na Gravidez: Uma Revisão
}

\author{
Eating Disorders in Pregnancy: A Review
}

\author{
Jordana Pinto Cardoso* \& António Pazo Pires \\ Instituto Superior de Psicologia Aplicada, Lisboa, Portugal
}

\begin{abstract}
Resumo
Esta revisão de literatura visa abordar os processos envolvidos nas perturbações do comportamento alimentar no período gravídico, nomeadamente no que respeita às vivências subjectivas das mulheres grávidas e dos seus companheiros. Apesar da escassez de estudos sobre a sua experiência neste período, sobretudo relativos às vivências dos intervenientes, é visível a variedade de respostas das grávidas que tiveram alguma perturbação do comportamento alimentar na sua história de vida. Para além da prevalência e dos riscos associados a estas perturbações para a grávida e para o bebé, analisa-se a precipitação, melhoria, manutenção ou agravamento de sintomas associados a estas perturbações e possíveis factores envolvidos. Aborda-se, finalmente, a importância na avaliação e intervenção dos técnicos de saúde.
\end{abstract}

Palavras-chave: Perturbações alimentares, gravidez, casal, parentalidade.

\begin{abstract}
This literature review aims at approaching the processes involved in eating disorders during pregnancy period, in particular regarding the subjective experiences of pregnant women and their partners. Despite the shortage of studies on their experience in this period, particularly concerning the experiences of stakeholders, there's a large variety of responses of pregnant women who had some eating disorder in their lives. In addition to the prevalence and risks associated to such disruptions for the women and their babies, we also analyze the precipitation, improvements, maintenance or worsening of symptoms related to eating disorders and other possible factors involved. It is also approached the importance of assessment and intervention on the part of health professionals.

Keywords: Eating disorders, pregnancy, couple, parenthood.
\end{abstract}

As perturbações do comportamento alimentar são psicopatologias que afectam sobretudo indivíduos do sexo feminino, maioritariamente jovens. Hoek e Hoeken (2003) estimam que, nos países desenvolvidos, uma em cada 100 mulheres serão diagnosticadas com uma perturbação do comportamento alimentar ao longo da sua vida, podendo aumentar até cinco vezes mais se se considerarem os sintomas que não preenchem todos os critérios de diagnóstico (Newton \& Chizawsky, 2006). Na maior parte dos casos, os indivíduos que procuram tratamento para uma perturbação do comportamento alimentar não preenchem então os critérios necessários para o diagnóstico de perturbações deste tipo, adoptando as perturbações do comportamento alimentar sem outra especificação o diagnóstico mais comum nos estudos de prevalência (Machado, Machado, Gonçalves, \& Hoek, 2007; Ward, 2008). Fairburn e Harrison (2003) apontam também a tendência para os pacientes passarem por mais do que uma catego-

\footnotetext{
* Endereço para correspondência: Instituto Superior de Psicologia Aplicada, Rua Jardim do Tabaco, 34, Lisboa, Portugal, 1149-041. E-mail: jcardoso@ispa.pt.
}

ria de diagnóstico ao longo da sua vida. Assim, alguns autores (Fairburn \& Bohn, 2005; Fairburn \& Harrison, 2003; Fairburn et al., 2007) referenciam a necessidade de questionar a validade das categorias diagnósticas actuais, com mais características em comum do que diferenciais.

Com a revolução industrial e a evolução do século XX, o papel da mulher sofreu modificações a diversos níveis, nomeadamente no que diz respeito à sua posição na família e no mercado de trabalho. Numa sociedade em constante mudança também o homem, consequentemente, viu um conjunto de transformações tomar lugar no seu quotidiano e nas suas experiências de vida. A própria parentalidade começa a focar-se cada vez mais não só na mulher mas também no homem e nas suas vivências enquanto pais, procurando dar ênfase às suas experiências pessoais, rompendo com as ideias assentes nos papéis parentais tradicionais.

Sendo a gravidez considerada como um período de transição de extrema importância para o casal, podem surgir factores que o vêm desafiar para uma posição ainda mais exigente, como é o caso da gravidez associada ao diagnóstico de uma perturbação do comportamento alimen- 
tar. Apesar de existirem diversas revisões de literatura sobre a gravidez, e muitas outras sobre as perturbações do comportamento alimentar, existem poucos estudos sobre as perturbações do comportamento alimentar na gravidez, sobretudo na abordagem ao casal, e uma grande escassez de investigações respeitantes às vivências subjectivas da gravidez associada a perturbações do comportamento alimentar.

Pretende-se assim abordar os processos envolvidos nas perturbações do comportamento alimentar no período gravídico, nomeadamente no que respeita às vivências subjectivas das mulheres grávidas e dos seus companheiros. Para além da prevalência e dos riscos associados a estas perturbações para a grávida e para o bebé, analisase a precipitação, melhoria, manutenção ou agravamento de sintomas associados a estas perturbações e possíveis factores envolvidos, bem como a importância na avaliação e intervenção dos técnicos de saúde.

\section{Perturbações do Comportamento Alimentar e Gravidez}

A gravidez caracteriza-se por alterações que requerem ajustamentos físicos, psicológicos e sociais. Ao engravidar, a mulher experiencia diversas transformações sobre as quais não tem controlo, nomeadamente a aparência do seu corpo, bem como uma grande imprevisibilidade (Larsson \& Andersson-Ellström, 2003; Mitchell-Gieleghem, Mittelstaedt, \& Bulik, 2002). Assim, se a gravidez já é um período de transição importante e de alterações significativas na vida do casal-expectante, a exigência é superior quando respeita a uma gravidez associada ao diagnóstico passado ou actual de perturbação do comportamento alimentar. É, então, pertinente o estudo da experiência das perturbações do comportamento alimentar no período gravídico, tendo em conta as mulheres com diagnóstico de perturbação do comportamento alimentar que engravidam, bem como as que desenvolvem estas perturbações durante a gravidez, no panorama da escassez de conhecimentos acerca da temática.

As perturbações do comportamento alimentar surgem com frequência nos anos férteis das mulheres. Ao mesmo tempo, verificam-se frequentemente alterações nas atitudes e comportamentos alimentares durante este período (Fahy \& O'Donoghue, 1991), mesmo em indivíduos sem perturbações alimentares, como as relatadas por Fairburn, Stein e Jones (1992), nomeadamente comer de forma saudável, comer em excesso, fortes aversões a comidas, bebidas ou cheiros específicos, ou os chamados "desejos" ou forte vontade de comer determinada comida ou bebida, ou cheirar determinada substância. Os acontecimentos de vida percebidos pelo indivíduo como stressantes podem também contribuir para a precipitação (Shmidt, Tiller, \& Treasure, 1993), recaída ou agravamento de perturbações do comportamento alimentar (Welch, Doll, \& Fairburn, 1997). A gravidez tem um impacto inevitável e complexo nas atitudes face ao peso e forma corporal e nos sintomas de perturbações do comportamento alimentar (Senior, Barnes, Emberson, \& Golding, 2005). A própria gravidez e a experiência da parentalidade podem ser temidas pelas suas consequências negativas como a falta de controlo sobre o peso e a própria vida (Striegel-Moore, 1994, citado por Tiller \& Treasure, 1998), impulsionando o ciclo de risco da perturbação.

Apesar de se saber, hoje em dia, que a fertilidade não está afectada e que existe possibilidade de engravidar, considerou-se durante algum tempo que a gravidez em mulheres com perturbações alimentares, sobretudo com anorexia nervosa, era rara, devido aos factores endocrinológicos, psicológicos e psicossociais associados à perturbação (Strimling, 1984; Weinfeld, Dubay, \& Burchell, 1977, citados por Bulik, Reba, Siega-Riz, \& ReichbornKjennerud, 2005). Morgan, Lacey e Sedgwick (1999) constataram inclusive uma elevada taxa de gravidez não planeada na bulimia nervosa já que as irregularidades menstruais eram erradamente associadas à infertilidade. No que respeita à anorexia nervosa, tal como afirmam Bonne, Rubinoff e Berry (1996), a gravidez é frequentemente detectada mais tarde do que nas outras mulheres.

Estudos demonstraram que a gravidez pode, por um lado, agravar, manter, reduzir, despoletar recaídas, ou mesmo evitar comportamentos de risco em mulheres com história passada ou actual de perturbações do comportamento alimentar, ou precipitar ou manter afastadas destes comportamentos as mulheres sem sintomatologia (Blais et al., 2000; Crow, Agras, Crosby, Halmi, \& Mitchell, 2008; Crow, Keel, Thuras, \& Mitchell, 2004; Dunker, Alvarenga, \& Alves, 2009; Edelstein, 2007; Hollifield \& Hobdy, 1990; Lemberg \& Phillips, 1989; Mitchell-Gieleghem et al., 2002; Newton \& Chizawsky, 2006; Ramchandani \& Whedon, 1988), podendo estas alterações ser permanentes ou transitórias (Blais et al., 2000; Newton \& Chizawsky, 2006). Siega-Riz et al. (2008) constataram que as mulheres com bulimia nervosa antes e durante a gravidez exibiam padrões dietéticos diferentes das grávidas sem perturbações do comportamento alimentar. Mulheres com episódios recentes de perturbação do comportamento alimentar parecem ter maior preocupação acerca do seu peso e forma corporal e mais comportamentos compensatórios (como utilização de laxantes, vómito auto-induzido e exercício físico) que as mulheres sem perturbação durante a gravidez (Micali, Treasure, \& Simonoff, 2007). A não-satisfação com o corpo pode igualmente melhorar (Davies \& Wardle, 1994) ou piorar com a gravidez (Crow et al., 2004).

Algumas mulheres pioram ou mesmo recomeçam estes comportamentos (Dunker et al., 2009; Edelstein, 2007; Kouba, Hälström, Lindholm, \& Hirschberg, 2005; Newton \& Chizawsky, 2006), sendo o principal impulsionador as alterações físicas e psicológicas que advêm da gravidez (Makino, Hashizume, \& Tsuboi, 2007), nomeadamente o medo relativo ao aumento de peso (Lemberg \& Phillips, 1989). Num estudo de Rand, Willis 
e Kuldau (1987), este medo surgiu com maior frequência no primeiro trimestre de gravidez e segundo mês do período pós-parto. Lingam e McCluskey (1996) revelam que a hiperemese gravídica pode também precipitar a bulimia nervosa em mulheres mais vulneráveis. Muitas destas mulheres experienciam medo de contribuir para o mal-estar do bebé (Lemberg \& Phillips, 1989; Lewis \& Grange, 1994), não prevenindo muitas vezes comportamentos de risco (Lewis \& Grange, 1994). O medo da perda de controlo é um medo muito comum nas mulheres com perturbações do comportamento alimentar neste período (Lemberg \& Phillips, 1989; Lewis \& Grange, 1994; Tiller \& Treasure, 1998), apesar de poder também surgir medo que a criança venha a desenvolver perturbações do comportamento alimentar, medo de não estar apta para tratar da criança ou o medo de perder a atenção do cônjuge (Lemberg \& Phillips, 1989). Bakan, Birmingham e Goldner (1991) sugerem que a gravidez e as complicações natais podem constituir-se enquanto factores de risco para a cronicidade na anorexia nervosa. Berg et al. (2008) revelaram que a incidência da bulimia nervosa no primeiro trimestre estaria associada a sintomas ansiosos e depressão, bem como baixa auto-estima e satisfação. Behar, González, Ariza e Aguirre (2008) indicam que as mulheres da sua amostra, grávidas com perturbações do comportamento alimentar, apresentam baixa auto-estima e dificuldades na identificação de sensações e emoções.

No entanto, muitas mulheres, como as relatadas no estudo de Madsen, Horder e Stoving (2009), apresentam remissão da perturbação do comportamento alimentar na gravidez com melhoria de sintomas, como dieta restritiva e vómito auto-induzido. Os autores apontam para possíveis causas psicológicas, sociais e endocrinológicas como factores de remissão da perturbação no período gravídico. Outros autores (Berg et al., 2008) associam a remissão de sintomas na gravidez a níveis elevados de satisfação e auto-estima. No entanto, os autores indicam que estes níveis elevados podem aumentar a probabilidade de remissão de bulimia nervosa na gravidez, mas também podem surgir como secundários à remissão, ou ainda serem influenciados por outros factores. Um estudo de Rocco et al. (2005) demonstra melhoria de sintomas a meio do período gravídico, mas retorno a níveis anteriores à gravidez no período pós-parto.

No período pós-parto, a maioria dos indivíduos com perturbações do comportamento alimentar tem, então, recaídas. Tendo em conta que, no período gravídico, muitas mulheres podem ingerir alimentos por preocupação com a saúde do bebé ou "comem por dois", o nascimento do bebé confronta estas mulheres com a anulação destas duas condicionantes, bem como com a parentalidade de um filho que, na maioria das vezes, não foi planeado, associado ao elevado risco de depressão pós-parto (Dunker et al., 2009; Edelstein, 2007; Mitchell-Gieleghem et al., 2002). Morgan et al. (1999), num estudo realizado com grávidas bulímicas, constataram que $57 \%$ das participantes tinham sintomas bulímicos mais acentuados que no período pré-parto, mas 34\% já não tinham o diagnóstico associado. Assim, sugerem quatro factores preditores de recaída em comportamentos bulímicos: comportamentos bulímicos persistentes durante a gravidez, história de anorexia nervosa antes do diagnóstico de bulimia nervosa, diabetes gestacional e gravidez não planeada. Num outro estudo (Lai, Tang, \& Tse, 2006) com uma amostra de indivíduos sem diagnóstico de perturbação do comportamento alimentar, os autores encontraram valores de perturbações alimentares duas vezes superiores nos seis meses pós-parto, sendo os preditores do estudo a falta de suporte do companheiro, fraca ligação mãe-bebé, perturbação alimentar durante a gravidez e depressão pós-parto. Também Crow et al. (2008) constataram que os episódios de compulsão alimentar, purgação e cognições de perturbação alimentar melhoram com a gravidez, apesar de tenderem a níveis pré-gravídicos no período pós-parto. Factores psicossociais como a ligação mãe-feto, o suporte do companheiro e o estado de saúde mental pós-parto podem estar associados à perturbação alimentar durante a transição da mulher para a maternidade, na medida em que, se a mulher tiver uma forte ligação ao feto, suporte por parte do companheiro e/ou saúde mental, tende a ter comportamentos mais saudáveis no período pós-parto e um melhor ajustamento na transição para a maternidade (Lai et al., 2006). Astrachan-Fletcher, Veldhuis, Lively, Fowler e Marcks (2008) referem que as exigências da maternidade e as preocupações com a imagem corporal intensificadas pelas alterações corporais da gravidez podem predispor as mulheres a uma exacerbação dos sintomas de perturbações do comportamento alimentar, bem como o desenvolvimento de perturbações do humor no período pós-parto.

Os estudos de prevalência de perturbações do comportamento alimentar na gravidez apontam para resultados de $0,1 \%$ para a anorexia nervosa, $0,7 \%$ a $8,4 \%$ para a bulimia nervosa e $0,1 \%$ a $23,4 \%$ para perturbações do comportamento alimentar sem outra especificação (Behar et al., 2008; Bulik et al., 2007; Lai et al., 2006; Torgersen et al., 2008; Turton, Hughes, Bolton, \& Sedgwick, 1999).

\section{Riscos Associados}

A literatura tem-se focado sobretudo nos riscos associados às perturbações do comportamento alimentar na gravidez, sem contemplar frequentemente os factores associados ou a experiência subjectiva das grávidas e seus companheiros. Apesar de, actualmente, já se saber que estas mulheres têm possibilidade de engravidar com frequência, sendo que a fertilidade não está comprometida, o decorrer saudável da gravidez parece comportar riscos significativos e alterações no seu bem-estar, assim como no dos fetos e mesmo no bem-estar das pessoas significativas que as rodeiam.

O baixo peso da mulher e/ou o ganho de peso inadequado durante a gravidez podem contribuir para o apare- 
cimento de anemia, o desenvolvimento de doença crónica (como doença cardiovascular), ou nascimento por cesariana (Barker et al., 1993; Bulik et al., 2005; Franko et al., 2001; James, 2001), chegando mesmo a existir relatos de morte associada ao diagnóstico de anorexia nervosa na gravidez (Ahmed, Balakrishnan, Minogue, Ryan, \& McKiernan, 1999). Os riscos incluem igualmente crescimento intra-uterino retardado, malformações congênitas, problemas no desenvolvimento do SNC, aborto espontâneo, nascimentos prematuros, nascimentos de nados-mortos, baixo peso à nascença, baixos valores do Índice de Apgar, ou fissura no palato ou lábio ou microcefalia (Bulik et al., 2005; Edelstein, 2007; James, 2001; Kouba et al., 2005; Lacey \& Smith, 1987; MitchellGieleghem et al., 2002; Newton \& Chizawsky, 2006; Stewart, 1992). Bebés prematuros e com baixo peso à nascença podem igualmente desenvolver problemas como hipoglicémia, hipotermia, risco aumentado de infecções, efeitos neurológicos adversos e morte (Kouba et al., 2005; Stewart, 1992). Sollid, Wisborg, Hjort e Secher (2004) apontam que o risco de baixo peso à nascença, nascimentos prematuros e desenvolvimento retardado do feto é superior em mulheres com perturbações do comportamento alimentar. Estes riscos são mais frequentemente observados em indivíduos com diagnóstico de anorexia do que em indivíduos bulímicos (Bulik et al., 2005; Edelstein, 2007). Namir, Melman e Yager (1986) descobriram que a taxa de possíveis complicações na gravidez e de problemas no nascimento são iguais às da população não anoréctica quando as pacientes ganham o peso adequado ao longo da gravidez.

Com as frequentes recaídas no período pós-parto, em que as mulheres são responsáveis pela alimentação dos filhos, as interacções mãe-bebé podem ser prejudicadas pelo facto de não estarem preparadas e se sentirem sobrecarregadas pela responsabilidade da maternidade, bem como pelas suas ideias sobre peso e forma estarem distorcidas devido à sua perturbação, com consequências inclusive ao nível da alimentação do bebé (Dunker et al., 2009; Edelstein \& King, 1992; Lewis \& Grange, 1994). As mulheres com perturbação do comportamento alimentar tendem a amamentar os filhos por períodos de tempo mais curtos que as mulheres sem perturbação (Larsson \& Andersson-Ellström, 2003; Namir et al., 1986). Ao mesmo tempo, enquanto alguns autores alegam que a prematuridade, as complicações obstétricas e o baixo peso podem aumentar o risco de desenvolvimento de anorexia nervosa mais tarde, denominado o "ciclo de risco" (Bulik et al., 2005), outros autores consideram que episódios traumáticos no início de vida não parecem aumentar a susceptibilidade para o desenvolvimento de sintomatologia associada a perturbações do comportamento alimentar (Feingold, Sheir-Neiss, Melnychuk, Bachrach, \& Paul, 2002).

Os riscos para a mãe e para o bebé podem ser aumentados devido ao consumo de tabaco (Cliver et al.,1992) ou a outros factores de risco como os factores psicossociais já referidos por Lai et al. (2006), o que nos leva a inferir que o suporte social, nomeadamente o suporte prestado pelo companheiro, pode diminuir a taxa de riscos para a mãe e o bebé. $O$ factor com maior peso que Blais et al. (2000) encontraram no seu estudo, no que diz respeito ao momento do parto, foi o estatuto conjugal. Os autores descobriram que, na sua amostra, as mulheres casadas tiveram maior número de nascimentos-vivos que as mulheres solteiras. Também Lewis e Grange (1994) verificaram que as mulheres com perturbação do comportamento alimentar mais grave na gravidez foram as mesmas que se separaram ou divorciaram depois, o que levanta a hipótese de que as mulheres com percepção de relações estáveis estavam mais aptas a controlar os sintomas bulímicos durante a gravidez do que as que tendiam a ter problemas interpessoais, resultados que estão de acordo com outros autores (como Lacey \& Smith, 1987). Por outro lado, as mulheres podem sentir as relações conjugais como tendo efeitos negativos, nomeadamente no que respeita às suas inseguranças acerca da aparência e aos comentários dos cônjuges quanto às quantidades de comida ingerida (Namir et al., 1986).

As alterações de humor e sintomas afectivos na gravidez influenciados por perturbações alimentares estão escassamente descritos na literatura. As alterações endócrinas da gravidez e do puerpério podem estar associadas a sintomas depressivos e flutuações de humor (Tiller \& Treasure, 1998). Um estudo de Mazzeo et al. (2006) aponta que o perfeccionismo, traço observado com frequência em pacientes com perturbações alimentares, pode predispor indivíduos com estas perturbações a problemas de humor antes e depois do parto. A comorbilidade entre humor negativo e perturbações do comportamento alimentar revelam-se sobretudo numa visão negativa do futuro, ao mesmo tempo que os pacientes lutam com as estratégias de manutenção da magreza (Mazzeo et al., 2006). Shoebridge e Gowers (2000) revelam que existe maior ansiedade e preocupação durante a gravidez em mulheres com anorexia nervosa.

Após o nascimento do bebé, as mulheres com perturbação do comportamento alimentar presente ou no passado (inclusive as mulheres com remissão de sintomas na gravidez e no período pós-parto) apresentam um elevado risco de desenvolver depressão pós-parto (Abraham, 1998; Carter, McIntosh, Joyce, Frampton, \& Bulik, 2003; Franko et al., 2001; Morgan, Lacey, \& Chung, 2006; Morgan et al., 1999), tendo em conta que cerca de metade está já deprimida durante a gestação (Edelstein, 2007). Os valores de depressão pós-parto parecem ser, então, mais elevados em mulheres que têm ou tiveram perturbações de comportamento alimentar, quer tenham tido recaídas neste período ou tenham persistido com estes comportamentos no período gravídico (Mazzeo et al., 2006). No estudo realizado por estes autores, $60 \%$ das mulheres com bulimia nervosa e $40 \%$ das mulheres com anorexia nervosa desenvolveram depressão pós-parto. As alterações físicas e psicológicas do período gravídico parecem 
afectar não só a reincidência de sintomas no período gravídico mas também sintomatologia associada a depressão pós-parto (Makino et al., 2007). As mulheres com história de perturbação do comportamento alimentar demonstram igualmente níveis mais elevados de sintomatologia ansiosa na gravidez e no período pós-parto (Micali $\&$ Treasure, 2009).

\section{Técnicos de Saúde: Avaliação e Intervenção}

Os estudos indicam que o tratamento da anorexia nervosa durante a gravidez pode contribuir para a normalização do aumento de peso e, assim, para a diminuição dos riscos para os intervenientes (Namir et al., 1986). No entanto, os técnicos de saúde podem não conseguir identificar as perturbações do comportamento alimentar, já que (a) os pacientes apresentam relutância em revelar a sua problemática, mesmo quando o diagnóstico se refere à sua história passada (Edelstein \& King, 1992; Franko \& Spurell, 2000; Franko \& Walton, 1993; Lemberg \& Phillips, 1989; Mitchell-Gieleghem et al., 2002; Newton \& Chizawsky, 2006; Norre, Vandereycken, \& Gordts, 2001; Stewart, 1992), normalmente associado a sentimentos de vergonha (Newton \& Chizawsky, 2006); (b) muitas vezes, os sintomas mais visíveis, nomeadamente na anorexia nervosa (como é o caso da amenorreia), não estão presentes no período gravídico; (c) nem sempre as perturbações são evidenciadas pelo aspecto físico, como no caso da bulimia nervosa, que abrange indivíduos com qualquer peso (Newton \& Chizawsky, 2006); (d) outros sintomas podem esconder o diagnóstico (como a hiperemese gravídica, que pode esconder bulimia nervosa; Lingam \& McCluskey, 1996); e (e) as perturbações são egosintónicas, podendo traduzir-se em negação da perturbação (Newton \& Chizawsky, 2006). Além desta omissão frequente aos clínicos, as mulheres com perturbações do comportamento alimentar na gravidez podem inclusive esconder a perturbação ou tentar minimizar alguns comportamentos específicos aos cônjuges, familiares, amigos ou terapeuta.

Apesar de a maioria das mulheres ser pesada durante a gravidez, a maioria dos obstetras não questiona as grávidas acerca de sintomatologia depressiva, controlo do peso ou sintomas de perturbações do comportamento alimen$\operatorname{tar}$ (Abraham, 2001). Neste estudo, o autor indica ainda que os obstetras do sector privado apresentam menor tendência para questionar as mulheres acerca de possível historial de depressão e para referenciá-las a técnicos de saúde mental (Abraham, 2001). Surgem, então, exigências aos técnicos no sentido da importância da detecção precoce pelas equipas de cuidados primários (Manzato, Zanetti, \& Gualandi, 2009) e o desenvolvimento de estratégias de identificação e diagnóstico alternativas, como o acesso à história reprodutiva (nomeadamente aos ciclos menstruais) e psicossocial, a medição regular do peso, o exame físico (incluindo perturbações na mucosa oral, deterioração dos dentes, nitrogénio de ureia no sangue, entre outros) e questões específicas acerca de possíveis sintomas ("como se sente ao ser pesada todas as consultas?", "como se sente acerca do aumento de peso?", "como se sente com as alterações físicas na forma do seu corpo?") e estratégias utilizadas, dependendo das respostas fornecidas (Mitchell-Gieleghem et al., 2002; Newton \& Chizawsky, 2006). Quer no desenvolvimento como após a conclusão do diagnóstico, o indivíduo beneficia de uma intervenção ao nível multidisciplinar, inclusive no período pós-parto. Como afirmam Crow et al. (2008), a gravidez pode ser uma altura crucial para a identificação e tratamento de casos, nunca esquecendo que as pacientes com perturbações alimentares devem continuar a ser seguidas com frequência, mesmo depois da remissão, tendo em conta que o risco de recaída é muito grande. Propõe-se igualmente a intervenção dos técnicos no sentido da promoção do ajustamento dos pais neste período de grande exigência face às inúmeras alterações, nunca esquecendo que o papel do parceiro é essencial, quer como agente fundamental no diagnóstico e compreensão, quer como agente de suporte.

Porém, segundo Namir et al. (1986), as mulheres podem por si só utilizar diversas estratégias para lidar com a sua nova forma e o seu novo corpo, como alterações do foco da barriga para as coxas (onde ocorrem menos alterações), repetição de frases de encorajamento (como "isto não é gordura, é um bebé"), entre outras formas que definem a gravidez como prova de que são normais e de que recebem atenção do seu marido por estarem grávidas e não pela perturbação diagnosticada. A preocupação pelo bem-estar do feto parece ser a motivação principal para as alterações de comportamentos no sentido positivo (Dunker et al., 2009; Lemberg \& Pillips, 1989; Mitchell-Gieleghem et al., 2002). No entanto, a gravidez por si só pode surgir como impulsionadora de sentido de maturidade e responsabilidade (Lemberg \& Pillips, 1989), promovendo cognições e comportamentos saudáveis. $\mathrm{O}$ facto de poderem conceber pode restaurar sentimentos de normalidade, sentindo-se melhores e saudáveis (Namir et al., 1986). Segundo os mesmos autores, um outro benefício contemplado por mulheres grávidas com perturbações do comportamento alimentar é a percepção que podem ter com a satisfação dos cônjuges em relação à gravidez, recebendo igualmente maior atenção positiva dos mesmos, ao contrário da atenção negativa que recebiam pela perturbação do comportamento alimentar. Ao mesmo tempo pode também sentir-se "forçadas" a cuidar de si devido à saúde do feto.

\section{Conclusão}

Apesar da grande variedade de estudos acerca das perturbações do comportamento alimentar e acerca da gravidez, com a revisão de literatura realizada confirmouse a grande escassez de estudos relativos à experiência da perturbação do comportamento alimentar na gravidez, não tendo sido encontrados estudos específicos que abor- 
de esta temática no casal. Os estudos encontrados focamse sobretudo em factores quantitativos, como os riscos associados, e não na experiência propriamente dita da mulher ou do parceiro. Os estudos qualitativos encontrados foram sobretudo estudos de caso, incidentes apenas na problemática da mulher e, raramente, na sua experiência subjectiva. No que respeita à abordagem das perturbações, a maioria dos estudos incide simultaneamente nas duas principais perturbações (a anorexia nervosa e a bulimia), não as diferenciando na sua abordagem.

Considera-se, por isso, fundamental o estudo das experiências vivenciadas pela mulher e pelo seu parceiro quando se relaciona o período gravídico com uma perturbação do comportamento alimentar, tendo em conta que as duas situações em separado já são exigentes para o casal. Assim, estudos futuros acerca da temática deverão abordá-la de forma qualitativa, tentando compreender como a mulher e o parceiro são capazes de reflectir acerca da sua experiência subjectiva, como experienciam as mudanças corporais, como vivenciam a experiência do(a) parceiro(a), como se relacionam com a sua rede social e que estratégias de adaptação à situação utilizam.

\section{Referências}

Abraham, S. (1998). Sexuality and reproduction in bulimia nervosa patiens over 10 years. Journal of Psychosomatic Psychosomatic Research, 44, 491-502. Retrieved November 24, 2009, from EBSCOhost Academic Search Elite database.

Abraham, S. (2001). Obstetricians and maternal body weight and eating disorders during pregnancy. Journal of Psychosomatic Obstetrics \& Gynecology, 22(3), 159-163. Retrieved November 24, 2009, from Web of Knowledge.

Ahmed, S., Balakrishnan, V., Minogue, M., Ryan, C., \& McKiernan, J. (1999). Sudden maternal death in pregnancy complicated by anorexia nervosa. Journal of Obstetrics and Gynaecology, 19(5), 529-531. Retrieved February 3, 2009 , from EBSCOhost Academic Search Elite database.

Astrachan-Fletcher, E., Veldhuis, C., Lively, N., Fowler, C., \& Marcks, B. (2008). The reciprocal effects of eating disorders and the postpartum period: A review of the literature and recommendations for clinical care. Journal of Women's Health, 17(2), 227-239. Retrieved November 24, 2009, from Web of Knowledge.

Bakan, R., Birmingham, C., \& Goldner, E. (1991). Chronicity in anorexia nervosa: Pregnancy and birth complications as risk factors. International Journal of Eating Disorders, 10(6), 631-645. Retrieved February 3, 2009, from EBSCOhost Academic Search Elite database.

Barker, D., Gluckman, P., Godfrey, K., Harding, K., Owens, J., \& Robinson, J. (1993). Fetal nutrition and cardiovascular disease in adult life. Lancet, 341, 938-941. Retrieved November 17, 2008, from EBSCOhost Academic Search Elite database

Behar, R., González, J., Ariza, M., \& Aguirre, A. (2008). Transtornos de la conducta alimentaria en mujeres embarazadas controladas en atención primaria. Revista Chilena de Obstetricia y Ginecología, 73(3), 155-162. Retrieved October 14, 2009, from SciELO.
Berg, C., Bulik, C., Holle, A., Torgersen, L., Hamer, R., Sullivan, P., et al. (2008). Psychosocial factors associated with broadly defined bulimia nervosa during early pregnancy: Findings from the Norwegian mother and child cohort study. Australian and New Zealand Journal of Psychiatry, 42, 396-404. Retrieved November 25, 2009, from EBSCOhost Academic Search Elite database.

Blais, M., Becker, A., Burwell, R., Flores, A., Nussbaum, K., Greenwood, D., et al. (2000). Pregnancy: Outcome and impact on symptomatology in a cohort of eating-disordered women. International Journal of Eating Disorders, 27, 140-149. Retrieved October 29, 2008, através da fonte EBSCOhost Academic Search Elite database.

Bonne, O., Rubinoff, B., \& Berry, E. (1996). Delayed detection od pregnancy in patients with anorexia nervosa: Two case reports. International Journal of Eating Disorders, 20(4), 423-425. Retrieved February 3, 2009, from EBSCOhost Academic Search Elite database.

Bulik, C., Holle, A., Hamer, R., Berg, C., Torgersen, L., Magnus, P., et al. (2007). Patterns of remission, continuation and incidence of broadly defined eating disorders during early pregnancy in the norwegian mother and child cohort study (MoBa). Psychological Medicine, 37(8), 1109-1118. Retrieved November 17, 2008, from EBSCOhost Academic Search Elite database.

Bulik, C., Reba, L., Siega-Riz, A., \& Reichborn-Kjennerud, T. (2005). Anorexia nervosa: Definition, epidemiology, and cycle of risk. The International Journal of Eating Disorders, 37, 2-9. Retrieved November 17, 2008, from Wiley InterScience.

Carter, F., McIntosh, V., Joyce, P., Frampton, C., \& Bulik, C. (2003). Bulimia nervosa, childbirth, and psychopathology. Journal of Psychosomatic Research, 55, 357-361. Retrieved November 24, 2009, from EBSCOhost Academic Search Elite database.

Cliver, S., Goldenberg, R., Cutter, G., Hoffman, H., Copper, R., Gotlieb, S., et al. (1992). The relationships among psychosocial profile, maternal size, and smoking in predicting fetal growth retardation. Obstetrics And Gynecology, 80(2), 262-267. Retrieved October 29, 2008, from EBSCOhost Academic Search Elite database.

Crow, S., Agras, W., Crosby, R., Halmi, K., \& Mitchell, J. (2008). Eating disorder symptoms in pregnancy: A prospective study. The International Journal of Eating Disorders, 41(3), 277-279. Retrieved October 29, 2008, from EBSCOhost Academic Search Elite database.

Crow, S., Keel, P., Thuras, P., \& Mitchell, J. (2004). Bulimia symptoms and other risk behaviors during pregnancy in women with bulimia nervosa. International Journal of Eating Disorders, 36, 220-223. Retrieved November 6, 2008, from EBSCOhost Academic Search Elite database.

Davies, K., \& Wardle, J. (1994). Body image and dieting in pregnancy. Journal of Psychosomatic Research, 38(8), 787799. Retrieved December 2, 2008, from EBSCOhost Academic Search Elite database.

Dunker, K., Alvarenga, M., \& Alves, V. (2009). Transtornos alimentares e gestação - Uma revisão. Jornal Brasileiro de Psiquiatria, 58(1), 60-68. Retrieved October 14, 2009, from SciELO.

Edelstein, C. (2007). Eating disorders and pregnancy: An overview. Women's Health in Primary Care, 10, 32-44. Retrieved October 29, 2008, from EBSCOhost Academic Search Elite database. 
Edelstein, C., \& King (1992). Pregnancy and eating disorders. In J. Yager (Ed.), Special problems in managing eating disorders (pp. 163-184). Washington, DC: American Psychiatric Association. Retrieved October 29, 2008, from EBSCOhost Academic Search Elite database.

Fahy, T., \& O'Donoghue, G. (1991). Eating disorders in pregnancy. Psychological Medicine, 21(3), 577-580. Retrieved November 25, 2009, from EBSCOhost Academic Search Elite database.

Fairburn, C., \& Bohn, K. (2005). Eating disorder NOS (EDNOS): An example of the troublesome 'not otherwise specified' (NOS) category in DSM-IV. Behaviour Research and Therapy, 43(6), 691-701. Retrieved November 10, 2008, from EBSCOhost Academic Search Elite database.

Fairburn, C., Cooper, Z., Bohn, K., O’Connor, M., Doll, H., \& Palmer, R. (2007). The severity and status of eating disorder NOS: Implications for the DSM-V. Behaviour Research and Therapy, 45, 1705-1715. Retrieved November 23, 2009, from ScienceDirect.

Fairburn, C., \& Harrison, P. (2003). Eating disorders. The Lancet, 361, 407-416. Retrieved November 23, 2009, from EBSCOhost Academic Search Elite database.

Fairburn, C., Stein, A., \& Jones, R. (1992). Eating habits and eating disorders during pregnancy. Psychosomatic Medicine, 54, 665-672.

Feingold, E., Sheir-Neiss, G., Melnychuk, J., Bachrach, S., \& Paul, D. (2002). Eating disorder symptomatology is not associated with pregnancy and perinatal complications in a cohort of adolescents who were born preterm. International Journal of Eating Disorders, 31, 202-209. Retrieved February 3, 2009, from EBSCOhost Academic Search Elite database.

Franko, D., Blais, M., Becker, A., Delinsky, S., Greenwood, D., Flores, A., et al. (2001). Pregnancy complications and neonatal outcomes in women with eating disorders. The American Journal of Psychiatry, 158, 1461-1466. Retrieved November 24, 2008, from EBSCOhost Academic Search Elite database.

Franko, D., \& Spurell, E. (2000). Detection and management of eating disorders during pregnancy. Obstetrics \& Gynecology, 95, 942-946. Retrieved December 2, 2008, from EBSCOhost Academic Search Elite database.

Franko, D., \& Walton, B. (1993). Pregnancy and eating disorders: A review and clinical implications. International Journal of Eating Disorders, 13(1), 41-48. Retrieved October 29, 2008, from EBSCOhost Academic Search Elite database.

Hoek, H., \& Hoeken, D. (2003). Review of the prevalence and incidence of eating disorders. The International Journal of Eating Disorders, 34, 383-396. Retrieved November 18, 2008, from EBSCOhost Academic Search Elite database.

Hollifield, J., \& Hobdy, J. (1990). The course of pregnancy complicated by bulimia. Psychotherapy, 27(2), 249-255. Retrieved February 3, 2009, from EBSCOhost Academic Search Elite database.

James, D. (2001). Eating disorders, fertility, and pregnancy: Relationships and complications. Journal of Perinatal and Neonatal Nursing, 15(2), 36-48. Retrieved February 3, 2009, from EBSCOhost Academic Search Elite database.

Kouba, S., Hälström, T., Lindholm, C., \& Hirschberg, A. (2005). Pregnancy and neonatal outcomes in women with eating disorders. Obstetrics \& Gynecology, 105(2), 255-260. Retrieved November 24, 2008, from GreenJournal.
Lacey, J., \& Smith, G. (1987). Bulimia nervosa: The impact of pregnancy on mother and baby. British Journal of Psychiatry, 150, 777-781. Retrieved November 18, 2008, from EBSCOhost Academic Search Elite database.

Lai, B., Tang, C., \& Tse, W. (2006). A longitudinal study investigating disordered eating during the transition to motherhood among Chinese women in Hong Kong. International Journal of Eating Disorders, 39(4), 303-311. Retrieved November 24, 2008, from EBSCOhost Academic Search Elite database.

Larsson, G., \& Andersson-Ellström, A. (2003). Experiences of pregnancy-related body shape changes and of breast-feeding in women with a history of eating disorders. European Eating Disorders Review, 11, 116-124. Retrieved November 24, 2008, from EBSCOhost Academic Search Elite database.

Lemberg, R., \& Phillips, J. (1989). The impact of pregnancy on anorexia nervosa and bulimia. International Journal of Eating Disorders, 8(3), 285-295. Retrieved October 29, 2008 , from EBSCOhost Academic Search Elite database.

Lewis, L., \& Grange, D. (1994). The experience and impact of pregnancy in bulimia nervosa: A series of case studies. European Eating Disorders Review, 2(2), 93-105. Retrieved October 29, 2008, from EBSCOhost Academic Search Elite database.

Lingam, R., \& McCluskey, S. (1996). Eating disorders associated with hyperemesis gravidarum. Journal of Psychosomatic Research, 40(3), 231-234. Retrieved October 29, 2008, from EBSCOhost Academic Search Elite database.

Machado, P., Machado, B., Gonçalves, S., \& Hoek, H. (2007). The prevalence of eating disorders not otherwise specified. The International Journal of Eating Disorders, 40, 212-217. Retrieved November 18, 2008, from EBSCOhost Academic Search Elite database.

Madsen, I., Horder, K., \& Stoving, R. (2009). Remission of eating disorders during pregnancy: Five cases and brief clinical review. Journal of Psychosomatic Obstetrics and Gynecology, 30(2), 122-126. Retrieved November 24, 2009, from Web of Knowledge.

Makino, M., Hashizume, M., \& Tsuboi, K. (2007). Effects of pregnancy on eating disorders. Journal of Psychosomatic Obstetrics and Gynecology, 147-150.

Manzato, E., Zanetti, T., \& Gualandi, M. (2009). Pregnancy in severe anorexia nervosa. International Journal of Eating Disorders, 42(1), 84-86. Retrieved November 24, 2009, from Web of Knowledge.

Mazzeo, S., Landt, M., Jones, I., Mitchell, K., Kendler, K., Neale, M., et al. (2006). Associations among postpartum depression, eating disorders, and perfectionism in a population-based sample of adult women. International Journal of Eating Disorders, 39, 202-211. Retrieved November 24, 2008, from EBSCOhost Academic Search Elite database.

Micali, N., \& Treasure, J. (2009). Biological effects of a maternal ED on pregnancy and foetal development: A review. European Eating Disorders Review, 17, 448-454. Retrieved November 24, 2009, from EBSCOhost Academic Search Elite database.

Micali, N., Treasure, J., \& Simonoff, E. (2007). Eating disorders symptoms in pregnancy: A longitudinal study of women with recent and past eating disorders and obesity. Journal of Psychosomatic Research, 53(3), 297-303. Retrieved November 25, 2009, from Web of Knowledge. 
Mitchell-Gieleghem, A., Mittelstaedt, M., \& Bulik, C. (2002). Eating disorders and childbearing: Concealment and consequences. Birth, 29(3), 182-191. Retrieved November 24, 2008, from EBSCOhost Academic Search Elite database.

Morgan, J., Lacey, J., \& Chung, E. (2006). Risk of postnatal depression, miscarriage, and preterm birth in bulimia nervosa: Retrospective controlled study. Psychosomatic Medicine, 68, 487-492. Retrieved November 24, 2009, from EBSCOhost Academic Search Elite database.

Morgan, J., Lacey, H., \& Sedgwick, P. (1999). Impact of pregnancy on bulimia nervosa. British Journal of Psychiatry, 174, 135-140. Retrieved November 24, 2008, from EBSCOhost Academic Search Elite database.

Namir, S., Melman, K., \& Yager, J. (1986). Pregnancy in restricter-type anorexia nervosa: A study of six women. The International Journal of Eating Disorders, 5(5), 837-845. Retrieved October 29, 2008, from EBSCOhost Academic Search Elite database.

Newton, M., \& Chizawsky, L. (2006). Treating vulnerable populations: The case of eating disorders during pregnancy. Journal of Psychosomatic Obstetrics and Gynecology, 27(1), 5-7. Retrieved October 29, 2008, from EBSCOhost Academic Search Elite database.

Norre, J., Vandereycken, W., \& Gordts, S. (2001). The management of eating disorders in a fertility clinic: Clinical guidelines. Journal of Psychosomatic Obstetrics and Gynaecology, 22(2), 77-81. Retrieved December 2, 2008, from EBSCOhost Academic Search Elite database.

Ramchandani, D., \& Whedon, B. (1988). The effect of pregnancy on bulimia. International Journal of Eating Disorders, 7(6), 845-848. Retrieved February 3, 2009, from EBSCOhost Academic Search Elite database.

Rand, C., Willis, D., \& Kuldau, J. (1987). Pregnancy after anorexia nervosa. International Journal of Eating Disorders, 6(5), 671-674. Retrieved February 3, 2009, from EBSCOhost Academic Search Elite database.

Rocco, P., Orbitello, B., Perini, L., Pera, V., Ciano, R., \& Balestrieri, M. (2005). Effects of pregnancy on eating attitudes and disorders: A prospective study. Journal of Psychosomatic Research, 59(3), 175-179. Retrieved November 24, 2009, from Web of Knowledge.

Shmidt, U., Tiller, J., \& Treasure, J. (1993). Psychosocial factors in the origins of bulimia nervosa. International Review of Psychiatry, 5, 51-59. Retrieved November 24, 2008, from EBSCOhost Academic Search Elite database.

Senior, R., Barnes, J., Emberson, J., \& Golding, J. (2005). Early experiences and their relationship to maternal eating disorder symptoms both lifetime and during pregnancy. British Journal of Psychiatry, 187, 268-273. Retrieved November 25, 2008, from EBSCOhost Academic Search Elite database.

Shoebridge, P., \& Gowers, S. (2000). Parental high concern and adolescent-onset anorexia nervosa: A case-control study to investigate direction of causality. British Journal of Psychiatry, 176(2), 132-137. Retrieved December 16, 2008, from EBSCOhost Academic Search Elite database.

Siega-Riz, A., Haugen, M., Meltzer, H., Holle, A., Hamer, R., Torgersen, L., et al. (2008). Nutrient and food group intakes of women with and without bulimia nervosa and binge eating disorder during pregnancy. American Journal of Clinical Nutrition, 87(5), 1346-1355. Retrieved November 24, 2009, from Web of Knowledge.
Sollid, C., Wisborg, K., Hjort, J., \& Secher, N. (2004). Eating disorder that was diagnosed before pregnancy and pregnancy outcome. American Journal of Obstetrics and Gynecology, 190(1), 206-210. Retrieved November 24, 2009, from Web of Knowledge.

Stewart, D. (1992). Reproductive functions in eating disorders. Annals of Medicine, 24(4), 287-291. Retrieved December 2, 2008, from EBSCOhost Academic Search Elite database.

Tiller, J., \& Treasure, J. (1998). Eating disorders precipitated by pregnancy. European Eating Disorders Review, 6, 178187. Retrieved October 29, 2008, from EBSCOhost Academic Search Elite database.

Torgersen, L., Holle, A., Reichborn-Kjennerud, T., Berg, C., Hamer, R., Sullivan, P., et al. (2008). Nausea and vomiting of pregnancy in women with bulimia nervosa and eating disorders not otherwise specified. International Journal of Eating Disorders, 41(8), 772-727. Retrieved November 24, 2009, from EBSCOhost Academic Search Elite database.

Turton, P., Hughes, P., Bolton, H., \& Sedgwick, P. (1999). Incidence and demographic correlates of eating disorder symptoms in a pregnant population. International Journal of Eating Disorders, 26(4), 448-452. Retrieved November 24, 2009, from Web of Knowledge.

Ward, V. (2008). Eating disorders in pregnancy. British Medical Journal, 336, 93-96. Retrieved November 24, 2009, from Web of Knowledge.

Welch, S., Doll, H., \& Fairburn, C. (1997). Life events and the onset of bulimia nervosa: A controlled study. Psychological Medicine, 27, 515-522. Retrieved November 24, 2008, from EBSCOhost Academic Search Elite database.
Recebido: 03/05/2010 $1^{a}$ revisão: 30/07/2010 $2^{a}$ revisão: $03 / 11 / 2010$ Aceite final: $12 / 11 / 2010$ 